\title{
La ceremonia de entrega de los Premios Princesa de Asturias y la teoría de las relaciones públicas: la organización de actos como técnica de gestión de la comunicación en las instituciones.
}

\author{
The award ceremony of the Princess of Asturias Awards and the \\ theory of public relations: the organization of acts as a \\ management technique for communication in institutions.
}

\author{
Marta Pulido Polo ${ }^{1}$ \\ Universidad de Sevilla \\ martapulido@us.es
}

Recepción: 22.11.2016 Revisión: 27.12.2016 Aceptación: 27.12.2016 Publicación: 15.01.2017

\section{Resumen (máximo 300 palabras)}

Las relaciones públicas deben concebirse como un proceso de gestión de las relaciones al servicio de la gestión de la comunicación de las corporaciones y las instituciones. En este contexto, el objetivo principal de este trabajo es analizar la Ceremonia de entrega de los Premios Princesa de Asturias como una técnica específica de relaciones públicas institucionales orientada a la legitimación y notoriedad de la Casa Real y los sucesivos Herederos a la Corona ante la opinión pública nacional e internacional, trasladando la imagen de continuidad y normalidad democrática. Para lograr la consecución de este objetivo principal, este trabajo realiza una revisión teórica sustentada en el uso de fuentes de datos secundarios, fundamentalmente bibliográficos y archivísticos. Los resultados evidencian utilidad de la organización de actos como herramienta de gestión de la opinión pública para vehicular y difundir los mensajes institucionales pertinentes en cada momento histórico.

Palabras claves: Ceremonia, Premios Princesa de Asturias, relaciones públicas, opinión pública

\footnotetext{
${ }^{1}$ Consultora de Relaciones Públicas. Profesora Acreditada Contratada Doctora, trabaja como Asociada en la Universidad de Sevilla. Doctora por la Universidad de Sevilla. Técnico en Comunicación Empresarial y Protocolo. Directora de la Consultora la bicicleta de Design \& Communication. Directora de Comunicación de la Organización Internacional de Ceremonial y Protocolo (OICP). Presidenta de la Asociación de Relaciones Públicas y Protocolo de Andalucía (ARPPA). Miembro del Grupo de investigación en Relaciones Públicas, Ceremonial y Protocolo LAUREA Hispalis. Miembro del Grupo de Investigación Historia del Pensamiento Jurídico-Político (GIHPJ-P). martapulido@us.es.
} 


\section{Abstract (maximun 300 words)}

Public relations should be conceived as a process of managing relationships at the service of communication management of corporations and institutions. In this context, the main objective of this work is to analyze the Ceremony of delivery of the Prizes Princess of Asturias as a specific technique of institutional public relations oriented to the legitimation and notoriety of the Royal House and the successive Heirs to the Crown before the, national and international, public opinion, transferring the image of continuity and democratic normality. In order to achieve this main objective, this work makes a theoretical revision based on the use of secondary data sources, mainly bibliographic and archival. The results show usefulness of the organization of acts as a tool for public opinion management to disseminate and disseminate relevant institutional messages in each historical moment.

Keywords: Ceremony, Princess of Asturias Awards, public relations, public opinion

\section{Sumario}

1. Introducción

2. Desarrollo

3. Conclusiones

4. Bibliografía

\section{Summary}

1. Introduction

2. Exposition

3. Conclusion

4. Bibliography

\section{Introducción}

La Fundación Príncipe de Asturias se constituye en 1980 ligando la figura del Príncipe Heredero a la Corona de España a valores nobles ligados a la actualidad social de cada época desde su creación tales como la solidaridad, las ciencias, las artes, etc.

Solo un año más tarde después de su creación, en 1981, se celebra ya la primera ceremonia de entrega de los entonces recién creados Premios Príncipe de Asturias, hecho que significa la gran importancia que la ceremonia de entrega tiene para el desarrollo de la propia Fundación (en adelante FPA) como acontecimiento institucional revestido de ceremonia orientado a la relevancia y notoriedad pública.

En su primera edición existían seis categorías que, con el paso del tiempo se han ido incrementando en consonancia a la evolución de los tiempos y los intereses y realidades, primero de la sociedad española y, posteriormente, de la sociedad internacional. Las seis categorías de premios iniciales eran Premios a las Artes, Comunicación y Humanidades, Ciencias Sociales, Investigación Científica y Técnica, Letras y Cooperación Internacional. En 1986 se incorpora el Premio Príncipe de Asturias a la Concordia y un año más tarde el del Deporte, configurándose así la relación actual vigente de ocho categorías. 
Curiosamente, la Ceremonia de Entrega de los Premios Príncipe de Asturias, ha servido además como estrategia de internacionalización para de la figura del Heredero o Heredera a la Corona de España. De hecho, en sus inicios eran habituales los premiados "domésticos". Además, hasta 1999, los galardones se concedían a personalidades hispanoparlantes y, a partir de este año se internacionalizan más allá del mundo iberoamericano con el premio Príncipe de Asturias a las Letras concedido a Günter Grass a quien una obra literaria de creación y ensayo, y una trayectoria cívica y humana convierten en figura excepcional de la literatura, del humanismo crítico y del compromiso moral de nuestro tiempo. En octubre de 2014 el patronato de la Fundación Príncipe de Asturias aprobó que tanto la institución como los premios pasaran a denominarse "Princesa de Asturias», en alusión a la heredera de la Corona, la princesa Leonor de Borbón, como consecuencia de la Proclamación del Rey Felipe VI.

En este contexto, resulta evidente el interés que, desde el punto de vista relacional, tiene la ceremonia de entrega de los Premios Princesa de Asturias como estrategia de gestión de las relaciones para la situación de un mensaje de difusión, legitimidad y continuidad de la Casa Real ante la opinión pública tanto española e internacional.

Bajo este prisma, la pertinencia del presente estudio queda justificada en sí mismo, dada la ausencia más absoluta de estudios serios de corte científico que aborden este acto desde las ciencias sociales en general, y desde la teoría de las relaciones públicas en institucionales en particular.

\section{Objetivos y metodología}

En un sistema democrático bajo la forma de una monarquía parlamentaria, como es el caso español, uno de los mensajes clave que las instituciones están obligadas a posicionar en la opinión pública, en aras de gestionar sus relaciones públicas y legitimarse así ante sus entornos internos y externos, es el de normalidad democrática y el correcto ejercicio y aplicación de los preceptos legales, institucionales y morales a los que se suscriben los poderes e instituciones públicas.

En este contexto, la organización de actos se concibe como una de las técnicas más efectivas para gestionar la percepción pública de las instituciones. Bajo estos postulados relacionistas, el objetivo principal de este trabajo es analizar la Ceremonia de entrega de los Premios Princesa de Asturias como una técnica específica de relaciones públicas institucionales orientada a la legitimación y notoriedad de la Casa Real y los sucesivos Herederos a la Corona ante la opinión pública nacional e internacional, trasladando la imagen de continuidad y normalidad democrática. Para desarrollar este objetivo principal, es necesaria la consecución paulatina de los siguientes objetivos secundarios:

OS1: Introducir la funcionalidad de las ceremonias de entrega de los Premios Princesa de Asturias como una de las principales actividades de la FPA.

OS2: Construir un marco teórico sólido que circunscriba la organización de actos como una técnica específica de relaciones públicas institucionales

OS3: Vincular la organización de actos a la teoría de los modelos históricos tradicionales de relaciones públicas expuestos por Grunig y Hunt (2000). 
OS4: Delimitar la evolución histórica del concepto de opinión pública.

\section{Marco teórico: La organización de actos y las relaciones públicas institucionales.}

\subsection{Los cuatro modelos de Grunig y Hunt.}

Basándose en el análisis del desarrollo histórico de su ejercicio profesional, Grunig introduce cuatro modelos conductuales de las relaciones públicas que representan los valores, fines y comportamientos de las organizaciones cuando practican las relaciones públicas (Xifra, 2003:53).

Tal y como explican Grunig y Hunt (2000: 72-73):

Hemos elegido el término "modelo" para describir las cuatro clases de Relaciones Públicas que pensamos que han evolucionado a lo largo de su historia, a fin de subrayar que se trata de abstracciones. Cuando se emplea científicamente, un modelo es una abstracción de la realidad. (...) Así pues, hemos elegido construir cuatro modelos de Relaciones Públicas que pesamos que ayudarán a entender su historia (la de las Relaciones Públicas formales) y a comprender la manera en que se practican hoy en día.

Una vez establecidos, Grunig y Hunt (2000: 74-103) realizan un análisis de cada uno de los modelos atendiendo en cada uno de ellos el objeto o intención, la naturaleza de la comunicación, los modelos de comunicación, la naturaleza de la investigación y su origen histórico. En este sentido, en la tabla 16, el lector puede observar la tabla resumen que estos autores realizan describiendo cada uno de los cuatro modelos en función de las características analizadas, a las que se añade dónde se practican en la actualidad y el porcentaje estimado de las organizaciones que ejercen cada uno de los modelos (Grunig y Hunt, 2000: 73).

Para Otero (1998, 2000, 2009), estos cuatro modelos tienen su antecedente clásico más lejano en Gorgias de Platón (387 a.C.) en el que:

(...) aparecen cuatro personajes clave_Gorgias, Polo, Cacicles y Sócrates_y cada uno de ellos realiza una exposición sobre su visión de la retórica desde diferentes postulados que coinciden con los citados modelos. (...) el debate sobre la utilización de la comunicación persuasiva en las relaciones públicas, convierte a los primitivos sofistas en antecesores remotos de los relacionistas públicos de ayer y aún de hoy (...) (Otero, 2009: 25-26).

Los cuatro modelos giran en torno al eje formado por dos dimensiones dicotómicas: dirección (unidireccionalidad vs. bidireccionalidad) y equilibrio o no de los efectos o finalidad perseguidos (asimétrico vs. simétrico) (Xifra, 2003: 53 y Arceo Vacas, 2004: 94). Tomando como punto de partida las aportaciones de James y Larissa Grunig (1992: 285-321), Arceo (2004) describe la dirección y el propósito como los elementos vertebradores de los cuatro modelos.

En primer lugar, con respecto a la dirección, afirma Arceo (2004: 94): 
La dirección delimita si el modelo es unidireccional, es decir, si solo ofrece información de uno de los actores comprometidos (del agente de prensa o del informador público que lleva a cabo tal acción o acciones en concreto), o por el contrario, si es bidireccional, brindando la oportunidad del diálogo y del intercambio de la información entre los actores comprometidos (el emisor y los receptores). Concretamente, los Grunig (1992: 289) hacen las siguientes afirmaciones sobre la variable dirección: La comunicación unidireccional difunde la información; es un monólogo. La comunicación bidireccional intercambia información; constituye un diálogo.

Es decir, la primera variable definitoria de los cuatro modelos es la direccionalidad de la comunicación que se erige como monólogo en el caso de la unidireccionalidad y diálogo en el caso de la bidireccionalidad.

Atendiendo a esta perspectiva dialógica, es reseñable la aportación de Xifra (2003: 106-125) que será tratada en mayor profundidad en el epígrafe centrado en la perspectiva relacional de las relaciones públicas por cuanto centra principalmente su atención en el concepto de relación con una orientación dialógica. En este sentido, Xifra (2003: 108) sustrae, de la literatura concerniente al concepto de diálogo desde la perspectiva de la comunicación, la filosofía y la psicología, los cinco grandes rasgos del dialogismo para la articulación de una teoría del diálogo en relaciones públicas. De esta forma, los atributos de mutualidad, proximidad, empatía, riesgo y compromiso constituyen, según el autor, los principios de dicha teoría.

En segundo lugar, el propósito como segundo de los elementos vertebradores de los modelos de actuación de relaciones públicas, explica si el modelo es asimétrico o simétrico (Arceo Vacas, 2004: 94). Desde la perspectiva de las relaciones públicas, la asimetría habla de una situación predominante de la organización frente a los públicos de la misma. Por su parte la simetría, hace referencia al equilibrio en la relación organización-públicos.

En este sentido afirma Arceo Vacas (2004:94):

En el caso de ser asimétrico, destaca el carácter dominante de la organización afectada, la cual intenta por todos los medios influir sobre la imagen, actitud, intención y comportamiento de los públicos parea hacer valer su posición ante ellos. Sin embargo, si el modelo es de corte simétrico, se puede hablar de un mayor equilibrio entre la organización y los públicos, ya que la empresa o entidad parece desprenderse de ese halo omnipotente que le llevaba a no conectar con éstos y a querer persuadirlos, sin más.

En definitiva, la mayor parte de los autores españoles consultados (Noguero, 1990 y 1995; Arceo Vacas, 1994 y 2004; Xifra, 2003; y Otero, 2000 y 2009) referencian, resumen y analizan los cuatro modelos históricos de Grunig, actualmente elevados a cinco y propuestos en distintas obras (Grunig y Hunt, 1984, 2000; Grunig 1992; Dozier, Grunig y Grunig, 1995) y que se han ido desarrollando y superponiendo a lo largo de la historia sin que desaparezca ninguno (Otero 2009: 25).

A continuación, se ofrece una breve descripción del planteamiento original de los cuatro modelos (Grunig y Hunt, 2000:30-32, 72-80), tomando como referencia, fundamentalmente, 
el objeto de las relaciones públicas, la naturaleza de la comunicación y la investigación en cada uno de ellos, para realizar una breve exposición final del quinto y último de los propuestos.

\subsubsection{El modelo agente de prensa.}

En este modelo, las relaciones públicas realizan una función de propagación de la información a través, fundamentalmente, de comunicaciones incompletas y a menudo falseadas, en la que además no hay posibilidad de feedback (comunicación unidireccional) (Grunig y Hunt, 2000: 74-75). La investigación es prácticamente nula, y se reduce a la observación informal o al recuento de si los materiales de publicity han sido utilizados en los medios de comunicación. A veces, puede incluir un control de los asistentes a un determinado acto o promoción (Grunig y Hunt, 2000: 77).

\subsubsection{El modelo de información pública.}

En el modelo de información pública el objeto de las relaciones públicas es la difusión de información, con carácter no necesariamente persuasivo (Grunig y Hunt, 2000: 74). Como en el modelo anterior, la comunicación es unidireccional aunque, a diferencia del anterior, este modelo considera la importancia de proporcionar la información completa, de decir la verdad (Grunig y Hunt, 2000: 75). En general, la investigación es escasa, reduciéndose a tests de legibilidad o de número total de lectores (Grunig y Hunt, 2000: 77).

\subsubsection{El modelo asimétrico bidireccional.}

En el modelo asimétrico bidireccional, el objeto de las relaciones públicas es la persuasión científica, que supone utilizar la teoría de las ciencias sociales e investigar las actitudes y conductas para persuadir a los públicos con el objetivo de que acepten el punto de vista de la organización y la apoyen (Grunig y Hunt, 2000: 74). La comunicación es, por tanto, bidireccional, con la particularidad de que en este modelo la comunicación se planifica cuidadosamente para conseguir modificar las conductas y actitudes del público (Grunig y Hunt, 2000: 75).

En los modelos bidireccionales, la investigación es esencial, pudiendo ser formativa (aquella que ayuda a planificar una acción y a elegir los objetivos) o evaluativa (aquella que permite descubrir si el objetivo se ha cumplido). En el modelo asimétrico bidireccional, el profesional de las relaciones públicas emplea la investigación formativa para conocer qué es lo que el público podría aceptar y tolerar, y la formativa para determinar cuáles han sido los efectos del programa o campaña (Grunig y Hunt, 2000: 77-78).

\subsubsection{El modelo simétrico bidireccional.}

En este modelo, las relaciones públicas sirven de mediadores entre la organización y sus públicos, con el objetivo de conseguir una comprensión mutua entre ambos (Grunig y Hunt, 2000: 74). La naturaleza de la comunicación es pues bidireccional, consistiendo en un diálogo en el que, tanto la organización como sus públicos, sean susceptibles de modificar sus actitudes o conductas (Grunig y Hunt, 2000: 75). Para ello, es necesaria una investigación 
formativa que permita identificar cómo es percibida la organización por sus públicos y las consecuencias que tiene aquélla sobre éstos. Por su parte, la investigación evaluativa, en el modelo simétrico, mide si un esfuerzo de relaciones públicas ha mejorado realmente la comprensión entre la organización y sus públicos (Grunig y Hunt, 2000: 78).

En palabras de Otero (2009: 28):

Éste sería el único enfoque posible para las relaciones públicas de organizaciones "excelentes" y socialmente responsables que _desde la investigación_ no renuncian a sus objetivos persuasivos pero lo supeditan al mutuo entendimiento y al consenso.

(...) La negociación entre los distintos sujetos implicados en un acto puede enfocarse desde estas perspectivas con mayores garantías de éxito, y la configuración de los espacios y tiempos comunes se ajusta mejor a las identidades corporativas.

Tabla 1. Características de los cuatro modelos de relaciones públicas.

Agente de pre

Asimétrico

Fuente: Grunig y Hunt (2000: 73)

\subsubsection{Una visión contingente de los cuatro modelos.}

Antes de proceder a explicar el quinto de los modelos, especial relieve entraña la visión contingente de los cuatro modelos que exponen Grunig y Hunt (2000: 104). A través de esta perspectiva contingente, estos autores responden a la cuestión de cuál de los cuatro modelos esbozados es el "correcto". Desde esta perspectiva, ninguna aproximación es siempre apropiada para todas las condiciones. Sea cual sea el mejor enfoque, depende de la naturaleza de la organización y del entorno en el que debe sobrevivir. (...) Todo depende... afirman Grunig y Hunt (2000: 104).

De este modo, la utilización de un modelo u otro, depende del entorno en el que se englobe la organización puesto que, tal y como señalan Grunig y Hunt (2000: 173) la elección de un modelo de Relaciones Públicas debería ser contingente con la naturaleza del entorno de la organización.

El entorno puede ser estático o dinámico. En un entorno estático, la organización puede actuar sin prestar atención a los cambios, utilizando los modelos de agencia de prensa e información pública que no proporcionan feedback del exterior. Cuanto más dinámico sea el entorno, la organización debería optar por los modelos asimétrico bidireccional -que facilita el control de la organización de los cambios de su entorno- y el simétrico -que ayuda a la organización a cambiar el entorno, y además a modificar la organización cuando cambie el entorno (Grunig y Hunt, 2000: 173).

3.2. El quinto modelo, el antagonista cooperante. 
Con posterioridad a la elaboración de estos cuatro modelos, Grunig, junto con su esposa Larissa y David Dozier desarrollan, en 1995, un quinto modelo basado en la figura del profesional de las relaciones públicas. En este quinto modelo el responsable las relaciones públicas debe buscar y realizar los pactos necesarios para, respetando las discrepancias, centrarse en las coincidencias.

Como afirma Otero (2009: 28) este sería el modelo ideal para el ejercicio de las relaciones públicas contemporáneas ya que dota a los profesionales que las ejercen de una capacidad gestora y negociadora de gran valor para sus organizaciones por tres razones:

- Les ofrece una ventaja diferencial al ser capaces de prever los conflictos relacionales en potencia.

- Esta ventaja les ofrece una posibilidad proactiva para evitar la crisis. En caso de producirse, el relacionista público tendría en su mano la posibilidad de minimizar sus efectos sobre la organización.

- El amplio conocimiento sobre la cultura organizativa, posiciona al relacionista público en una posición privilegiada para comunicarla adecuadamente a sus públicos.

Capítulo 2. Marco conceptual

En este contexto cabe destacar que, según Otero (2000: 296 y 2009: 28) Arceo (1994: 20) planteaba ya hace unos años la elaboración de un quinto modelo, único enfoque posible para las relaciones públicas de organizaciones "excelentes", que tomaba como punto de partida el concepto del mutuo acuerdo del cuarto modelo pero asumiendo los propósitos de persuasión científica (desechando el enfoque de la presión sobre el público) del tercer modelo.

\subsection{La organización de actos y la gestión de la opinión pública}

Tal y como describe la perspectiva relacional (Ledingham y Brunnin, 1998; Ledingham, 2003, Ledingham, 2006) el concepto de relaciones públicas alude a la gestión que las organizaciones deben hacer de la amalgama de relaciones que la anclan a su entorno. Una correcta gestión del sistema relacional que se establece entre una organización y su entorno, permite a las organizaciones, sean empresas, sean instituciones, fortalecer todos aquellos sistemas de interconexión que la mantienen en una estabilidad (más o menos variable) en su contexto social, político y económico.

Para Xifra (2007 y 2011) las técnicas de relaciones públicas pueden dividirse en cuatro categorías básicas: las técnicas de relaciones públicas internas, las técnicas de relaciones con los medios de comunicación social, las técnicas de relación con la comunidad y las técnicas transversales. Dentro de las técnicas de gestión de las relaciones con la comunidad, una de las principales técnicas relacionistas es la organización de actos y eventos.

Bajo estos postulados, es fácil concluir que una de las técnicas más utilizadas y efectivas para gestionar las relaciones institución-opinión pública es la organización de actos.

Tal y como establece Otero (2000: 298-299), el concepto de opinión pública surge como tal en la Francia del siglo XVIII para aludir a la opinión del pueblo. De hecho, ya para los ilustrados del XVIII y los liberales del XIX, el concepto se va desarrollando para definir el 
resultado de la discusión pública de los grandes temas de interés para la sociedad. Posteriormente, Walter Lippmann (1889-1974), en su obra Public Opinión (1922) habla a cerca de la posibilidad de manipular la opinión pública para conseguir determinadas metas. En resumidas cuentas, la teoría de Lippman propugnaba que la cantidad de información que rodea al ser humano y la velocidad con la que se suceden los acontecimientos hacen que los ciudadanos corrientes no puedan tener un conocimiento pleno de todo lo que sucede todo el tiempo en todo el mundo, abandonándose y dejándose guiar por los eslóganes y flashes mentales que producen los medios de comunicación de masas.

Un año después, en 1923 Bernays publica el primer libro de relaciones públicas, Crystallizing Public Opinion, a través del que propugna que el asesor de relaciones públicas es el defensor de un punto de vista ante el público de una empresa o institución y que su profesión está en un estado de evolución cuyo futuro depende tanto de la creciente consciencia del público sobre la responsabilidad de los individuos, instituciones y organizaciones como de su propia consciencia de la importancia de su trabajo (Bernays, 1998: 46).

Del mismo modo, Bernays (1998: 46-47) sostiene que el asesor de relaciones públicas trabaja con un material vago y mal comprendido llamado opinión pública que se define como:

- Un término que describe un mal definido, variable e inestable grupo de juicios individuales.

- $\quad$ Un agregado final de opiniones individuales (uniformes o conflictivas entre sí) de los hombres y mujeres que constituyen una sociedad o un grupo social.

Bajo estas premisas, a través de la gestión de las relaciones con sus públicos, las organizaciones tratan de posicionar mensajes institucionales o corporativos que coadyuvan a la creación de un clima de entendimiento mutuo organización-públicos. En este proceso la intermediación de los medios de comunicación de masas es primordial para la difusión del mensaje ante la sociedad, ante la opinión pública.

Tal y como establece Castillo (2010: 159-184) esta situación social (estratégica) permite percibir a los mass media como receptores de todo tipo de actividades que persiguen poder incidir e influir sobre sus contenidos y temario, enmarcándose en una perspectiva que acrecienta su papel en una sociedad cada vez más comunicada. En este posicionamiento simbólico cada organización busca entablar un diálogo con la sociedad para poder explicar cuáles son sus objetivos y que pretende ofrecerle, todo ello en aras de la responsabilidad social de las organizaciones. Para ello es imprescindible contar con los medios de comunicación que son los que relacionan principalmente a las organizaciones con el conjunto de la sociedad y difunden sus mensajes con una finalidad claramente persuasiva.

\section{Análisis relacionista de la ceremonia de entrega de los premios Princesa de Asturias.}

Enmarcadas en las teorías expuestas, la ceremonia de entrega de os Premios Princesa de Asturias se erige como un objeto social e informativo capaz de gestionar la percepción pública de los Herederos al Trono de España a través de una institución externa, de carácter privado, que es la FPA, que nace ligada a valores elevados relacionados no solo con la élite nacional e internacional a nivel social, cultural, tecnológico, artístico, deportivo, solidario y 
científico, sino también relacionados con los más altos niveles de representación institucional que reflejan la estructura democrática y la forma política española.

Bajo esta premisa, cabe preguntarnos cuáles son los ítems tangibles a través de los cuales esta ceremonia objeto de estudio canaliza y vertebra los mensajes institucionales clave hacia la opinión pública.

Los resultados de nuestro estudio demuestran que los elementos clave a través de los que la Ceremonia de Entrega de los Premios Princesa de Asturias gestiona su percepción pública son: el emisor primario (real y percibido) del acto, los premiados, el ceremonial y protocolo y los asistentes principales.

\subsection{El emisor del acto.}

Dada la calidad y solemnidad percibida del acto en sí, un amplio sector de la opinión pública cree habitualmente que la Ceremonia de Entrega de los Premios Princesa de Asturias es un acto oficial, organizado por las instituciones públicas, del tipo del acto de conmemoración del 12 de octubre. Sin embargo, el organizador o emisor directo del acto es la Fundación Princesa de Asturias que se autodefine como una institución privada sin ánimo de lucro, cuyos objetivos son contribuir a la exaltación y promoción de cuantos valores científicos, culturales y humanísticos son patrimonio universal y consolidar los vínculos existentes entre el Principado de Asturias y el título que tradicionalmente ostentan los herederos de la Corona de España .

Entendemos pues que, desde la perspectiva de la gestión de la opinión pública, la ceremonia de entrega tiene un emisor "real" de carácter privado, la FPA, y un emisor "percibido" de carácter público y oficial que, además, redunda y coadyuva al posicionamiento de los valores de la Fundación analizada.

\subsection{Los premiados.}

Los resultados del análisis de la evolución año tras año de los premiados evidencian los aspectos que ya se adelantaban en la introducción de este trabajo, puesto que si bien los premiados de los primeros años son eminentemente nacionales, poco a poco se realiza una progresiva estrategia expansiva hacia Iberoamérica y finalmente, a partir de 1999, al resto de Europa y del mundo.

En todas las ceremonias de entregas de premios, los premiados parecen cubrir un doble rol: por un lado, el evidente protagonismo que merecen a la excelencia en el campo en el que se hallan insertos $y$, por otro lado, su papel como líderes de opinión y/o poder que ejercen una influencia directa entre sus públicos colaborando en la difusión de los mensajes que el emisor primario del acto persigue publicitar. De hecho es posible que el Nueva York, conocieran los Premios y a las instituciones vinculadas a los mismos, gracias a que en 2002 se le otorga el Premio Príncipe de Asturias de las Artes a Woody Allen.

\subsection{El ceremonial y el protocolo}


Una de las funciones principales del ceremonial y el protocolo como sistemas de comunicación persuasivos es precisamente, utilizar los espacios y los tiempos y todos los recursos de la comunicación verbal (discursos, agradecimientos, fallos del jurado, etc.) y no verbal (música ambiental, himnos, emblemas, escudos, banderas, presidencias y precedencias, etc.) en pos de engrandecer el acto y enfatizar el proceso de transmisión de los mensajes corporativos e institucionales que persiga el emisor primario o el organizador de la ceremonia.

Gracias a estos elementos, la organización de actos permite un incremento en la eficacia del mensaje y en la eficiencia del proceso de comunicación intencional que se genera a través del acto celebrado.

\subsection{Los asistentes principales.}

También la presencia de los asistentes principales y secundarios refuerzan los mensajes y valores corporativos e institucionales que se transmiten con la celebración de ceremonias de entrega y otros actos organizacionales. Resulta obvio que la asistencia de autoridades a actos empresariales no solo refuerza los vínculos existentes entre ambos sectores sociales sino que también lanzan mensajes estratégicos hacia la opinión pública.

En el caso concreto de la Ceremonia de Entrega de los Premios Princesa de Asturias, las primeras personalidades y autoridades del acto se vinculan con las altas instituciones del Estado, fundamentalmente con la Casa Real, la Jefatura del Estado y la figura de, desde 2014, la Heredera de la Corona, refrendando y ayudando a trasladar los valores fundacionales del organizador, la FPA.

\section{Conclusión final}

Los resultados evidencian utilidad de la organización de actos como herramienta de gestión de la opinión pública para vehicular y difundir los mensajes institucionales pertinentes en cada momento histórico. A través del uso estratégico de los cuatro elementos clave descritos: el emisor primario (real y percibido) del acto, los premiados, el ceremonial y protocolo y los asistentes principales, los actos permiten difundir mensajes corporativos e institucionales entre los públicos clave que componen la opinión pública.

Cabe tener en cuenta además, el poder de los medios de comunicación como intermediadores necesarios entre la organización y el posicionamiento masivo de los mensajes establecidos por el emisor primario u organizador del acto. De hecho, la solemnidad trasladada por el uso del ceremonial y el protocolo, la categoría de los premiados y la representación institucional al más alto nivel hacen del acto una referencia social de interés público tal que "obliga" a los medios de comunicación generales y específicos a hacerse eco del acto organizado.

\section{BIBLIOGRAFIA}

ARCEO VACAS, J.L. (2004). Las relaciones públicas en España. Madrid, Mc Graw Hill.

AREVALO, J. P. (2001). La Ciencia del Protocolo. Burgos, Ediciones Protocolo. 
AUSTIN, E. W. y PINKLETON, B. E. (2015). Strategic Public Relations Management: Planning and Managing Effective Communication Programs. New York, Taylor \& Francis.

BARQUERO CABRERO, J. D. y FERNÁNDEZ, F. (2004). El libro azul del protocolo y las relaciones públicas. McGraw-Hill / Interamericana de España, S.A.

BARQUERO CABRERO, J.D. Y FERNÁNDEZ, F. (2007). Los secretos del protocolo, las relaciones públicas y la publicidad. Valladolid, Lex Nova.

BENITO SACRISTÁN, P. (2008). Guía de estilo, protocolo y etiqueta en la empresa. Madrid, Wolters Kluwer España.

BERNAYS, E. L. (1998). Cristalizando la opinión pública. Barcelona, Gestión 2000.

CASTILLO, A. 2010. Introducción a las relaciones públicas. Ed. IIRP. Málaga.

COMESAÑA, P. 2012. "Las Relaciones Públicas como estrategia de comunicación en los eventos cinematográficos: los Premios Goya". Revista Internacional de Relaciones Públicas. Vol. II. №. 16: 113-130.

CUTLIP, S. M. y CENTER, A. H. 2001. Relaciones públicas eficaces. Editorial Gestión 2000. Barcelona.

GRUNIG, J. E. y HUNT, T. 2000. Dirección de relaciones públicas. Editorial Gestión 2000. Barcelona.

LEDINGHAM, J. A. (2003). "Explicating Relationship Management as a General Theory of Public Relations" en Journal of public relations research, 15 (2).

LEDINGHAM, J. A. (2006). "Relationship management: a general theory of public relations". Public Relations Theory II.LawrenceEarlbaum Associates, Inc. Mahwah, New Jersey.

LEDINGHAM, J. A. y BRUNING, S. D. (1998). "Relationship management in public relations: dimensions of an organization-public relationship" en Public Relations Review, Vol. 24, No. 1.

LOZANO BOTACHE, J. P. 2012. “Cine e interdisciplinariedad en la educación”. Libro de actas. I Congreso Virtual Internacional sobre Innovación Pedagógica y Praxis Educativa.

MARCOS RAMOS, M. 2010. “Alfabetización mediática. La educación en los medios de comunicación: cine formativo y televisión educativa". Teoría de la educación: Educación y Cultura en la Sociedad de la Información. Vol. 11. № 2: 303-321.

OTERO ALVARADO, M. T. 2000. Teoría y estructura del ceremonial y el protocolo. Mergablum edición y comunicación. Sevilla.

OTERO ALVARADO, M. T. 2006. "Relaciones públicas y gestión de públicos en eventos: los principios rectores del ceremonial y el protocolo". Anàlisi: Quaderns de comunicació i cultura. №. 34: 255-269. 
PULIDO POLO, M. (2012): “Técnicas de relaciones públicas en la comunicación organizacional" en Revista Internacional de Relaciones Públicas, vol. 3. Málaga, Instituto de Investigación en Relaciones Públicas.

PULIDO POLO, M. (2015): “Ceremonial y Protocolo: métodos y técnicas de investigación científica" en Opción, Especial nำ1, Venezuela.

RAMOS, F. (2002). La comunicación corporativa e institucional: de la imagen al protocolo. Madrid, Universitas.

RAMOS, F. (2003). Curso Superior de Comunicación y Protocolo. Vigo, Universidad de Vigo.

RAMOS, F. (2003). El protocolo universitario: historia, tradiciones y práctica actual del ceremonial en la universidad española. Vigo, Universidad de Vigo.

RAMOS, F. (2008). El protocolo de empresa: herramientas para crear valor. A Coruña, Netlibro.

SÁNCHEZ GONZÁLEZ, M.D.M. (2011). Fundamentos del ceremonial y el protocolo. Editorial Síntesis, Madrid.

SÁNCHEZ GONZÁLEZ, M.D.M., Gómez Requejo, M. V. y Pérez Margos, R. M. (2015). Historia del ceremonial y del protocolo. Madrid, Editorial Síntesis.

SEITEL, F. (2002). Teoría y práctica de las relaciones públicas. Madrid, Prentice Hall.

VÁZQUEZ SALLÉS, D. 2006. "La despensa: François Vatel. El espectáculo en la mesa de Luis XIV". Clío: Revista de historia. №. 62: 98-100.

VILLAFAÑE, J. (2004). La buena reputación. Claves del valor intangible de las empresas. Madrid, Pirámide.

WILCOX, D. L., AUTT, P. H., AGEE, W. K., y CAMERON, G.T. (2000). Relaciones públicas. Estrategias y tácticas. Madrid, Pearson Addison Wesley.

WILCOX, D. L., CAMERON, G.T. y XIFRA, J. J. (2012). Relaciones Públicas: Estrategias y tácticas. Madrid, Pearson.

WILCOX, D. L., CAMERON, G.T. y XIFRA, J. (2009). Relaciones públicas. Estrategias y tácticas. Madrid, Pearson Addison Wesley.

XIFRA, J. (2003). Teoría y estructura de las relaciones públicas. Madrid, Mc Graw Hill.

XIFRA, J. (2005). Planificación estratégica de relaciones públicas. Barcelona, Paidós.

XIFRA, J. (2007). Técnicas de las Relaciones Públicas. Barcelona, Editorial UOC.

XIFRA, J. (2010). Relaciones públicas, empresa y sociedad. Barcelona, UOC. 
XIFRA, J. (2011). Manual de relaciones públicas e institucionales. Madrid, Tecnos.

(C) (7) 